\title{
Development of genetic fuzzy logic controllers for complex production systems
}

\begin{abstract}
Complex production systems can produce more than one part type. For these systems, production rate and priority of production for each part type is determined by production controllers. In this paper, genetic fuzzy logic control (GFLC) methodology is used to develop two production control architectures namely ñgenetic distributed fuzzyò (GDF), and ñgenetic supervisory fuzzyò (GSF) controllers. Previously these controllers have been applied to single-part-type production systems. In the new approach the GDF and GSF controllers are developed to control complex production systems. The methodology is illustrated and evaluated using two test cases; two-part-type production line and re-entrant production systems. Genetic algorithm is used to tune the membership functions of input variables of GSF or GDF controllers. The objective function of the GSF controller minimizes the production cost based on work-in-process (WIP) and backlog costs, while surplus minimization is considered by GDF controller. The results show that GDF and GSF controllers can improve the performance of production systems. GSF controllers decrease the WIP level and its variations. GDF controllers show their abilities in reducing the backlog level but generally, production cost for GDF controller is greater than GSF controller.
\end{abstract}

Keyword: Genetic algorithm; Fuzzy logic controller; Genetic fuzzy logic controller; Complex production systems 